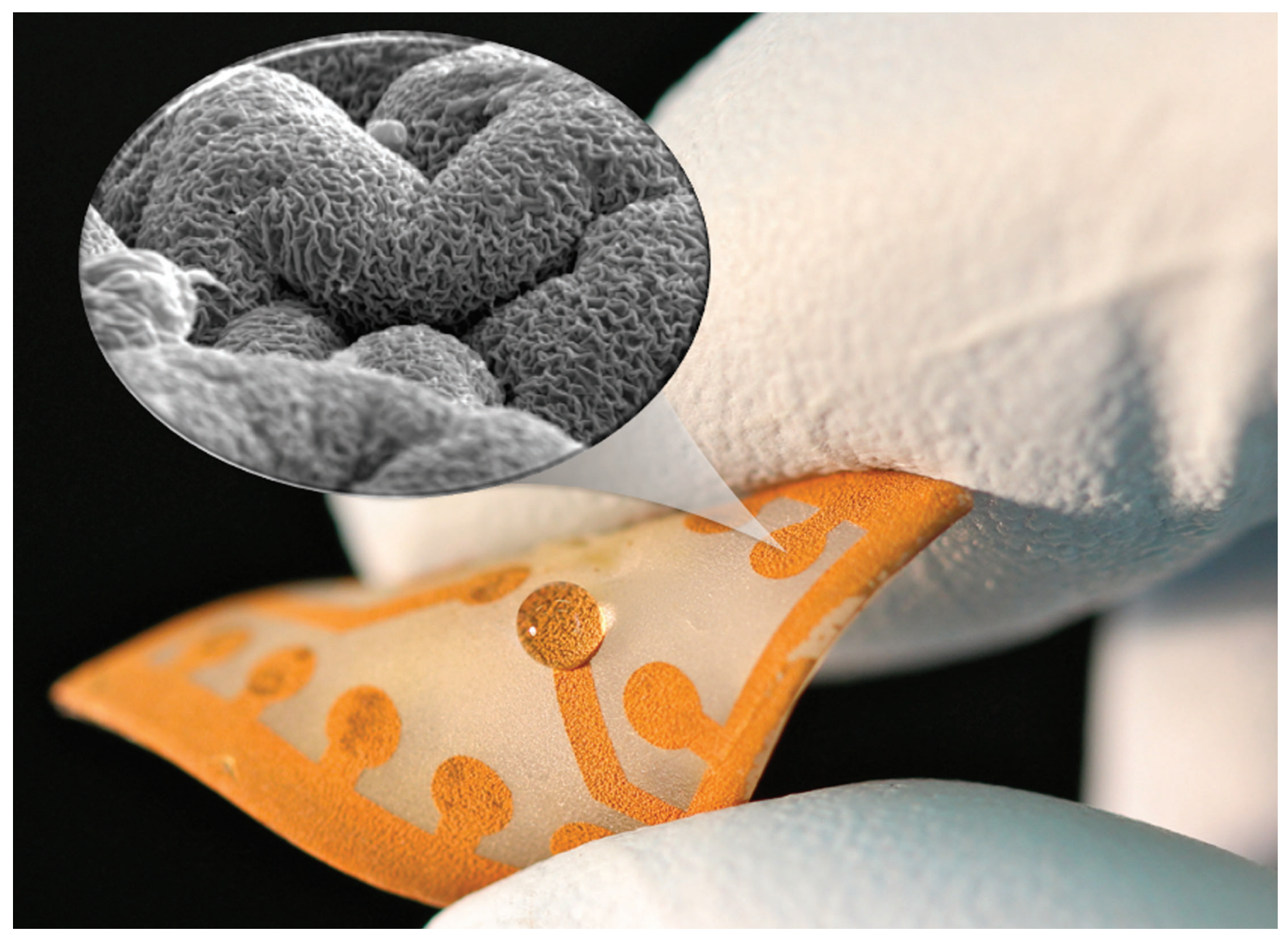

Featuring work from the group of Dr Michelle Khine at the Department of Biomedical Engineering, University of California, USA.

Title: Flexible shrink-induced high surface area electrodes for electrochemiluminescent sensing

In this work, photolithographically defined metallic thin film on commodity shrink-wrap is leveraged to create robust electrodes. By thermally shrinking the film, electrodes are reduced by $20 \times$ in footprint for improved resolution and conductivity with $>600 \%$ enhancements in electrochemically active surface area; as electrochemiluminescent sensors, they demonstrate improved limits of detection.

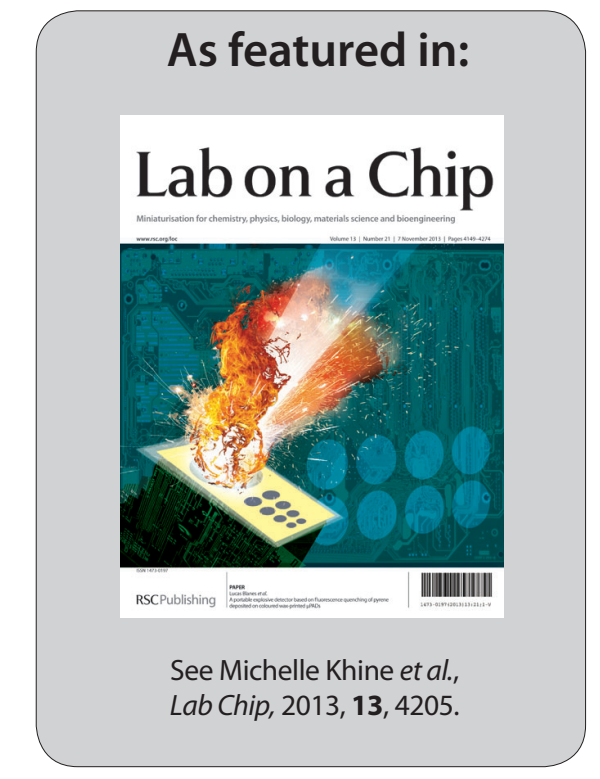


Cite this: Lab Chip, 2013, 13, 4205

\title{
Flexible shrink-induced high surface area electrodes for electrochemiluminescent sensing $\dagger$
}

\author{
Jonathan D. Pegan, ${ }^{\text {a }}$ Adrienne Y. Ho, ${ }^{\text {b Mark Bachman }}{ }^{\mathrm{c}}$ and Michelle Khine*a
}

Received 11th May 2013,

Accepted 6th August 2013

DOI: $10.1039 / c 31 c 50588 j$

www.rsc.org/loc

\begin{abstract}
Photolithographically defined metallic thin film on commodity shrink-wrap is leveraged to create robust electrodes. By thermally shrinking the film, electrodes are reduced by $20 x$ in footprint for improved resolution and conductivity with $>600 \%$ enhancements in electrochemically active surface area; as electrochemiluminescent sensors, they demonstrate improved limits of detection.
\end{abstract}

\section{Introduction}

Simple, robust, and affordable medical diagnostics that deliver rapid, sensitive results at the point of care (POC) would provide otherwise unavailable healthcare to developing regions. $^{1,2}$ Lab-on-a-chip (LOC) holds particular promise for this need with inherent advantages such as portability, rapid reaction times, and low required reagent volumes. ${ }^{1-4}$ However, there have been relatively few success stories of microfluidic devices being utilized in such regions to date. ${ }^{4,5}$ One of the fundamental barriers responsible for this is an over reliance on external, dedicated laboratory equipment needed to interface with many microfluidic devices. Microfluidic devices then become a component of an otherwise complex, expensive, non-portable system ill suited for the POC environment. ${ }^{1,4}$

While paper based microfluidics obviates these issues, they are inherently limited from detecting low concentrations, with typical detection ranges of $\mathrm{mM}$ to tens of $\mu \mathrm{M} .{ }^{6,7}$ Importantly, because colorimetric results are heavily influenced by ambient light these tests are limited in their quantitative ability and offer mostly qualitative, positive/negative conclusions.

Electrochemistry is an extremely sensitive mode of detection. Important biomolecule concentrations such as glucose, ${ }^{8,9}$ lactic acid, ${ }^{9}$ and uric acid ${ }^{10}$ have been successfully quantified at low concentrations, down to $\mathrm{fM}$, utilizing laboratory grade apparatus. Electrochemical detection schemes are also easily miniaturized making them attractive for POC applications. Electrochemiluminescence (ECL), where electro-generated species combine to convert electrochemical energy into radiative energy, has become increasingly relevant

${ }^{a}$ Department of Biomedical Engineering, University of California, Irvine, Irvine, CA 92697, USA. E-mail: mkhine@uci.edu

${ }^{b}$ Department of Chemical Engineering and Materials Science, University of California, Irvine, Irvine, CA 92697, USA

${ }^{c}$ Department of Electrical Engineering and Computer Science, University of

California, Irvine, Irvine, CA 92697, USA

† Electronic supplementary information (ESI) available. See DOI: 10.1039/ c3lc50588j for POC applications as it combines the advantages of both electrochemistry and chemiluminescence. Tris(2,2'-bipyridyl) ruthenium(II) $\left(\mathrm{Ru}(\mathrm{bpy})_{3}{ }^{2+}\right)$ and its many coreactants that react with the oxidized form of $\mathrm{Ru}(\mathrm{bpy})_{3}{ }^{2+}$ to emit light, is one of the most widely utilized ECL complexes in biomedical analytical devices. ${ }^{11-13}$ Biomolecules can be easily labeled with $\mathrm{Ru}(\mathrm{bpy})_{3}{ }^{2+}$ for immunoassay, magnetic bead, and DNA-based detection schemes. ${ }^{11} \mathrm{Ru}(\mathrm{bpy})_{3}{ }^{2+}$-based ECL has inherent advantages such as extreme stability, high sensitivity, low detection limit, wide dynamic range, ability to link with a wide range of analytes, excellent spatial and temporal control, good water solubility, and compatibility with separation techniques. Unlike colorimetric assays, ECL experiments are independent of ambient light. Delaney et al. were the first to show successful ECL detection of nicotinamide adenine dinucleotide (NADH) and 2-(dibutylamino)-ethanol (DBAE) in a microfluidic paper-based electrochemical device ( $\mu \mathrm{PED}) .{ }^{14}$ Importantly, this work also demonstrated good portability and compatibility with telemedicine, with ECL detection carried out using a cell phone camera. Ge et al. expanded the utility of ECL $\mu$ PEDs by developing a multiplexed sandwich type assay. ${ }^{7}$ Sardesai et al. recently showed microfluidic ECL with 10-25 fold enhancement in detection limits over nonmicrofluidic arrays. ${ }^{15}$ These aforementioned studies all represent important milestones in the development of ECL for POC.

We present an integrated, plastic ECL sensor using shapememory (shrink-wrap) polyolefin (PO) film. To date, these prestressed PO films have been used for creating many biomedical devices. ${ }^{3,16-19}$ This is the first demonstration of shrink electrode integration using a standard microfabrication approach. This method utilizes low cost, roll-to-roll compatible plastic and integration with well-established integrated circuit (IC) and microelectromechanical systems (MEMS) infrastructure; this enables a cost-effective, scalable manufacturing scheme capable of high resolution. Open-face design leveraging our superhydrophobic ( $\mathrm{SH}$ ) surfaces allows for easy spotting of reagents that remained confined to detection zones without the need for expensive external pumps. ${ }^{20}$ This surface 
tension controlled ability to manipulate liquid also lends itself to the possibility of multiplexing by integrating multiple samples on a single chip. Integration with microfluidics is also possible as we have previously demonstrated these thermoplastics are an excellent material for such applications. ${ }^{16,21}$ Importantly, samples can be easily reclaimed for subsequent analysis or storage. Inherent in shrink fabrication is the ability to create high surface area 'wrinkle' structures. ${ }^{22-24}$ When applied to metal thin films, high surface area electrodes can be simply fabricated, ${ }^{23,24}$ dramatically improving speed and performance of electrochemical sensors. ${ }^{25,26}$ To our knowledge, this is the first demonstration of combining photolithography and wet etching processes with shape-memory polymers for patterning electrodes. Importantly, with our added 20-fold increase in resolution from shrinking, we can create significantly higher resolution electrodes compared to photolithographic patterning alone as well as to previously published shrink-induced electrodes. ${ }^{23,24}$

\section{Experimental design}

\section{Fabrication of shrunk electrodes}

PO films (Sealed Air, Nexcel multilayer shrink film 955D) were affixed to $\mathrm{Si}$ wafers using $\mathrm{DIH}_{2} \mathrm{O}$ to provide mechanical support for compatibility with standard micromachining equipment. Characterization of the PO film properties and reproducibility have been previously reported. ${ }^{27} \mathrm{~A} 40 \mathrm{~nm} \mathrm{Au}$ thin film was deposited by electron-beam (e-beam) evaporation (Temescal CV-8). The Au coated PO film then underwent a photolithography and wet etching process for patterning of the desired electrode design (Fig. 1). In this two-part process Shipley 1827 photoresist (Microchem, MA) was patterned using a photomask and DUV flood hood (ABM, CA). After development of the sample, the remaining photoresist acted as a protective barrier against the $\mathrm{I}_{2}: \mathrm{KI}: \mathrm{H}_{2} \mathrm{O}(1: 4: 40) \mathrm{Au}$ etchant. The photoresist layer was removed using acetone. Patterned Au and PO films were then lifted from their Si wafer supports and heated past the PO glass transition temperature $\left(125{ }^{\circ} \mathrm{C}\right)$ using a commercial heat gun (Steinel HL1820S),

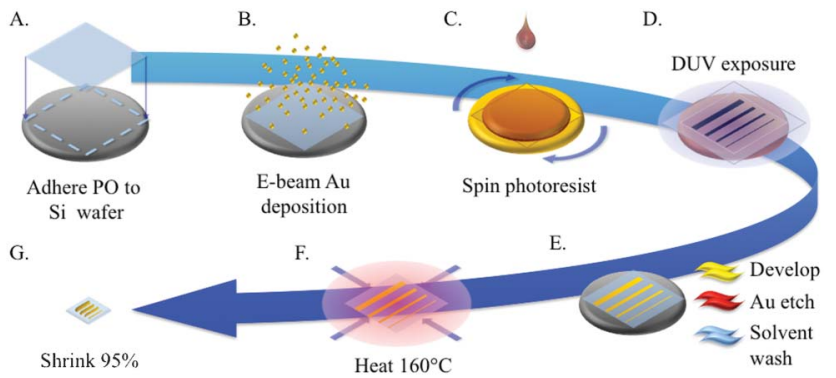

Fig. 1 Fabrication process flow. A. PO is adhered to a Si wafer support. B. $40 \mathrm{~nm}$ $\mathrm{Au}$ is e-beam deposited. C. Photoresist is spun onto the Au coated sample. D. The sample is exposed with a photomask to define electrode features. E. The sample is then developed, submerged in Au etchant, and cleaned with a solvent wash to produce Au patterned electrodes. F. The sample is then heated, G. to shrink the device footprint by $95 \%$.
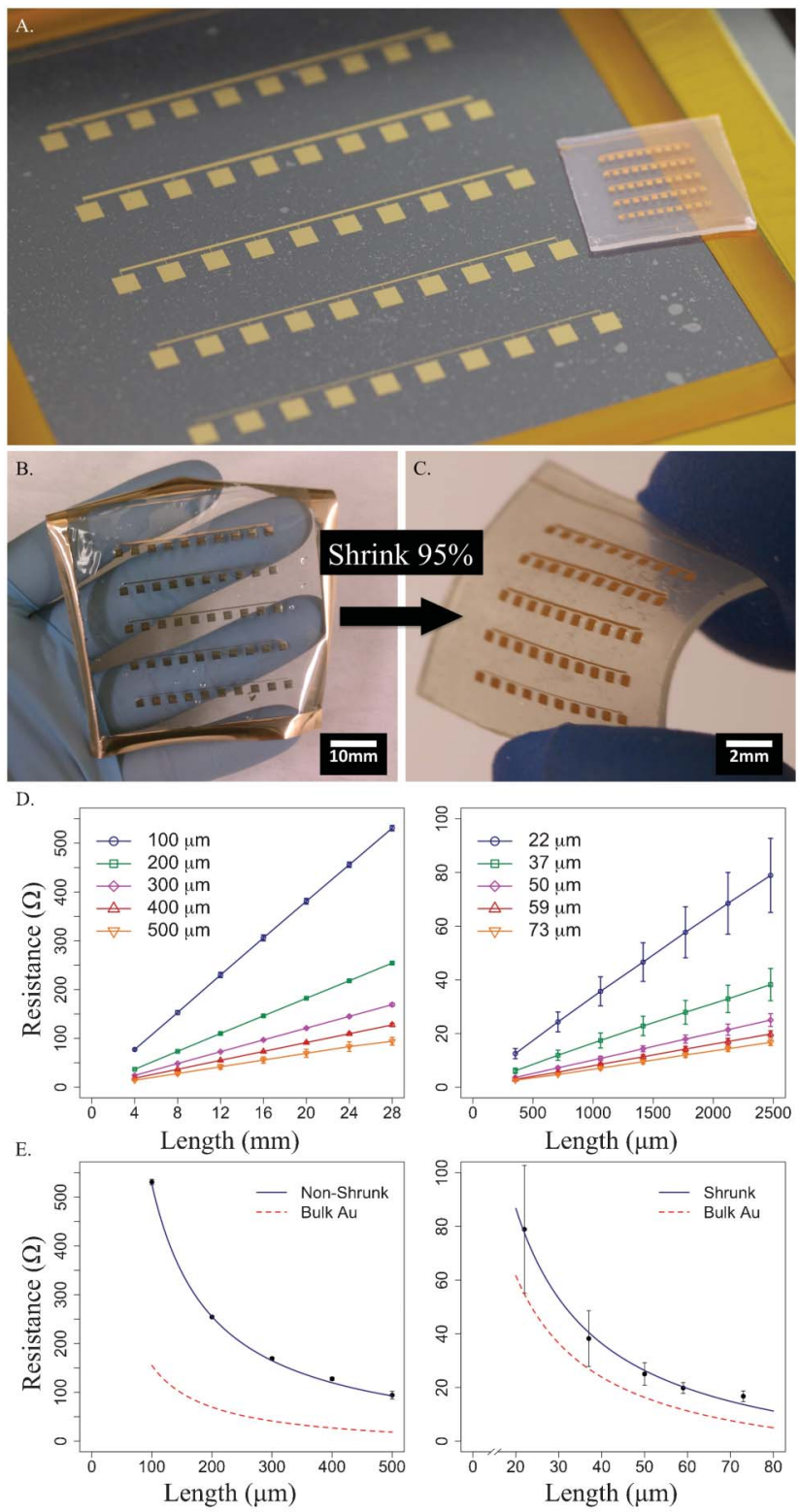

Fig. 2 Patterned Au thin film electrodes. A. Before (left) and after (right) thermal shrinking. B., C. Patterned electrodes remain flexible after the shrinking process. D. 4 pt measurements of non-shrunk (left) and shrunk (right) electrodes. E. Effective resistivity of the $40 \mathrm{~nm}$ thin film electrodes is decreased for shrunk electrodes, modeled with regression coefficient to correct systematic error from empirical measurements.

shrinking the patterned electrodes by $95 \%$ within seconds ${ }^{20,27}$ (Fig. 2A-C). Alternatively, wet etching can be avoided by patterning photoresist on the bare PO films prior to Au deposition with subsequent lift-off to create Au-free regions between electrodes. Otherwise, all processing steps remain the same.

\section{Electrode design}

Electrode designs were drawn using CAD software (Autodesk, Inc., CA). For designs that demanded higher resolution, CAD drawings were sent out to be printed on transparencies (Fineline Imaging, CO). Electrode line widths of $4 \mu \mathrm{m}$ were achieved after completing the thermal shrinking process using 
these printed Mylar photomasks. Alternatively, photomasks were laser machined in house from PMMA using a bench top laser cutter (VLS2.30, Universal Laser Systems, AZ). Utilizing the faster turn-around time of this method, devices could go from design to fabricated device within a few hours.

\section{Electrode characterization}

The electrical resistivity and electrochemically active surface area of the Au thin film electrodes were quantified before and after the shrinking process. A 4-point measurement method was performed to determine resistivity of the $\mathrm{Au}$ thin films. In short, a constant current is applied (E3611A, Agilent, CA) to individual $\mathrm{Au}$ thin film traces of different widths while voltage is recorded (117 Multimeter, Fluke, WA) to accurately measure resistance. Cyclic voltammetry was used to determine the electrochemically active surface area of the $\mathrm{Au}$ thin film electrodes as outlined by Gabardo et $a .^{23}$ Briefly, a threeelectrode electrochemical cell was setup with $\mathrm{Ag} / \mathrm{AgCl}$ reference electrode (SPE, TX), Pt counter electrode (Sigma Aldrich, $\mathrm{MO})$, and a $\mathrm{Au}$ thin film on PO serving as the working electrode. These three electrodes were submerged in $0.1 \mathrm{mM}$ $\mathrm{H}_{2} \mathrm{SO}_{4}$ (Sigma Aldrich, MO) while electric potential was cycled between $-0.1-1.5 \mathrm{~V}$ at a scan rate of $20 \mathrm{mV} \mathrm{s}^{-1}$ for 4 cycles (model DY2013 potentiostat). The electrochemical activity of the Au thin film electrodes was measured using the 4 th cycle.

\section{Methods and principles of ECL detection}

$\mathrm{Ru}(\mathrm{bpy})_{3}{ }^{2+}$ and DBAE (Sigma Aldrich, MO) coreactant was chosen as this system is well understood. ${ }^{28} 2 \mu \mathrm{L}$ mixtures of both reagents $(1: 1)$ were spotted onto 15 separate detection zones for each sensor. A dilution of DBAE $(5000-5 \mu \mathrm{M})$ was tested in triplicate on a single sensor while $\mathrm{Ru}(\mathrm{bpy})_{3}{ }^{2+}$ concentration $(5000 \mu \mathrm{M})$ was kept constant at each detection zone. All reagents for ECL were suspended in $0.1 \mathrm{mM}$ PBS (Sigma Aldrich, MO) unless otherwise stated. Patterned Au thin films constituted the working electrode while a transparent ITO coated glass microscope slide served as both the counter electrode as well as a top-sheet, enclosing the electrochemical cell when placed over the droplets. A PMMA spacer layer was used to hold the ITO counter electrode $1 \mathrm{~mm}$ above the ECL sensor so that contact was made with the droplet samples, but not the Au thin film electrodes. ECL was excited by applying $2.1 \mathrm{~V}$ from a bench top power source (E3611A, Agilent, CA). Notably, for POC applications a quick 1 $\mathrm{s}$ pulse from a battery could be used. Multiplexed ECL detection was carried out using a CMOS sensor from a consumer camera (Rebel Ti3, Canon, CA) positioned above the ITO counter electrode. Luminescent intensity was integrated over $1 \mathrm{~s}$ using the CMOS sensor at 1600 ISO sensitivity and the resulting images were analyzed using Image J software. ${ }^{29}$ Five different devices for each condition were tested.

\section{Results and discussion}

\section{Electrode characterization and performance}

Upon heating above the glass transition temperature $\left(T_{\mathrm{g}}\right)$ of PO, the stiffness mismatch between the two materials causes the Au thin film to buckle from the compressive stress of the shrinking polymer. ${ }^{23,24}$ Notably, robust integration of the $\mathrm{Au}$ thin film with the PO film occurs during this process (withstanding microfluidic flow, ${ }^{21}$ repeated molding ${ }^{30}$ without delamination). The PO partially reflows at high temperatures, increasing adhesion to the thin film without the need for adhesion layers between the polymer and Au. The resulting micro- and nano-wrinkling of the Au thin film is responsible for improved electrical performance, characterized by improved electrical resistivity.

4 pt measurements of non-shrunk and shrunk electrodes show that both have a linear decrease in resistance across patterned line electrodes of different widths (Fig. 2D). Measuring electrical resistivity before and after the thermal shrinking process shows a dramatic improvement of the wrinkled Au thin film electrodes $(0.033 \Omega \mu \mathrm{m})$ over the nonshrunk, planar Au electrodes $(0.077 \Omega \mu \mathrm{m})$. Notably, the effective resistivity of the $40 \mathrm{~nm}$ wrinkled $\mathrm{Au}$ thin film begins to approach bulk Au resistivity $(0.024 \Omega \mu \mathrm{m})$ (Fig. $2 \mathrm{E}$ ). This decrease in electrical impedance is therefore not simply an effect of reducing the mean path of resistance due to the smaller shape of the polymer. Cross-sections of the wrinkled $\mathrm{Au}$ thin films reveal many tens of micron-scale invaginations in the surface where adjacent wrinkles pack closely enough that they begin to coalesce, referred to as secondary folding. ${ }^{23}$ In a flat $\mathrm{Au}$ thin film, discontinuities produce a large effect in the resistivity of the film. ${ }^{31}$ We hypothesize that secondary folding in the wrinkled Au thin films creates an increase in electrical contacts thereby circumventing these discontinuities and reducing the effective resistivity of the wrinkled thin film electrodes.

Cyclic voltammetry comparing exposed $2 \mathrm{~mm} \times 2 \mathrm{~mm}$ areas of wrinkled verses flat $\mathrm{Au}$ thin film electrodes shows an improvement in electrochemically active surface area. Wrinkled Au thin film electrodes show a $647 \%$ enhancement as compared to flat thin films when integrating under the reduction peak of the CV diagrams (Fig. 3B). This enhancement is lower than the theoretically calculated surface area given the amount the PO film shrinks. We postulate this is likely the consequence of a high-degree of aforementioned secondary folding. This result is consistent across several wrinkled $\mathrm{Au}$ thin film devices suggesting reliable quality control between devices.

These hierarchical wrinkled structures (Fig. 3A), aside from increased electrical performance, exhibit a high degree of roughness that leads to interesting interactions with liquid samples. It has been previously shown that heterogeneous, rough structures create anti-wetting behavior. ${ }^{20,30}$ Additionally, creating large contrast between surface energies on a single substrate allows precise spatial control of small liquid volumes. ${ }^{20}$ The contrast between patterned $\mathrm{Au}$ thin films and exposed PO areas allows for the same effect. Previously, we have demonstrated that areas of contrasting surface energies yield very good spatial control and confinement of liquid droplets to specifically patterned regions. ${ }^{20}$ Here, we leverage the same ability to confine liquid droplets to patterned $\mathrm{Au}$ detection zones on wrinkled PO surfaces for parallel sensing of up to 15 individual samples in a device footprint of $1.5 \mathrm{~cm} \times 1.5 \mathrm{~cm}$ (Fig. 4). 

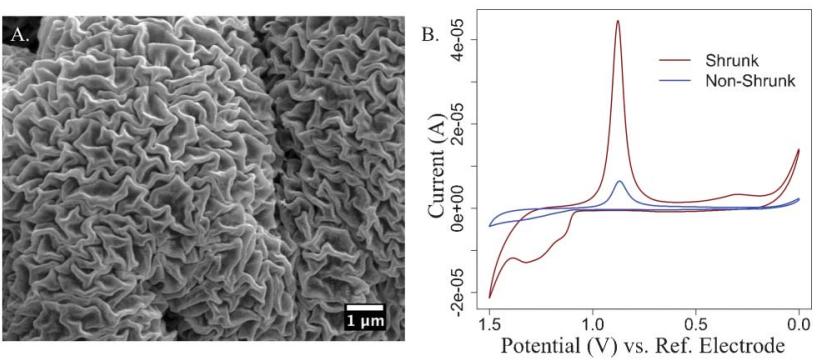

Fig. 3 High surface area wrinkled thin film electrodes. A. A high magnification SEM image shows the hierarchical electrode structure. B. Cyclic voltammetry shows $>600 \%$ increased electrochemically active surface area over non-shrunk, planar electrodes.

\section{Multiplexed ECL detection using digital camera}

In addition to creating wrinkled Au thin film electrodes, subtle differences in the fabrication process can be used to modify the structure of the PO film surface surrounding the electrodes. Using the method outlined in Fig. 1 results in a wrinkled PO surface as well as wrinkled thin film electrodes (Supplementary Fig. S1A $\dagger$ ). This is due to plasma generation during e-beam deposition which oxidizes the surface of the PO film. This oxide thin film acts as a stiffness layer much like the deposited Au thin film. ${ }^{19}$ This heterogeneous rough structuring increases the hydrophobicity of the polymer surface. The latter fabrication process described in the methods section leverages a photoresist lift-off process after Au deposition to protect the PO surface against oxidation. When Au patterned
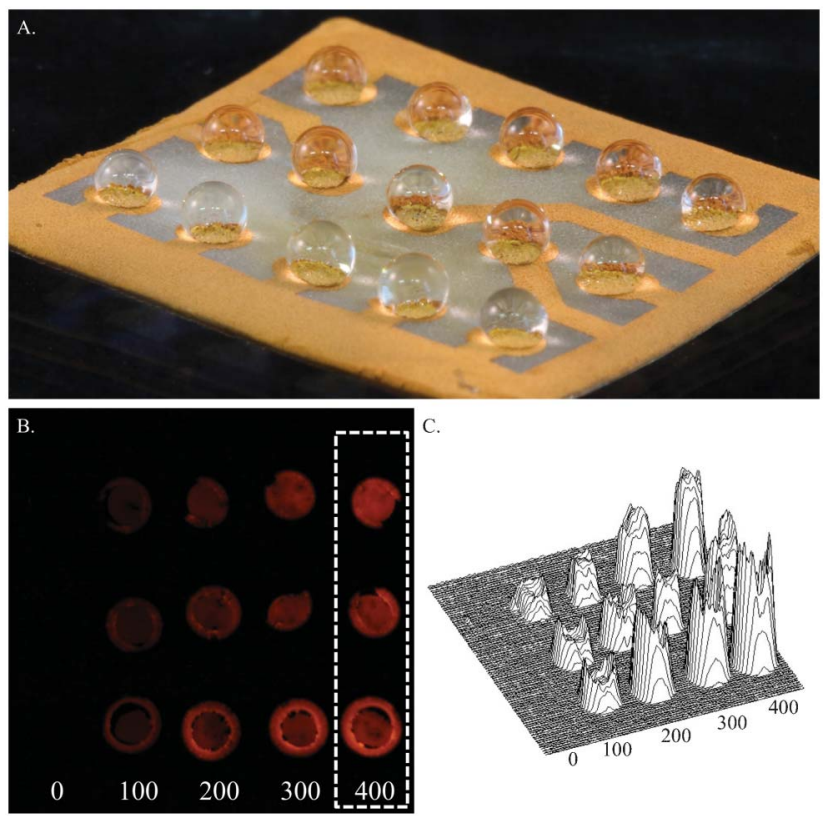

Fig. 4 Shrunk ECL sensor. A. ECL sensor with wrinkled Au thin film electrodes spotted with $2 \mu \mathrm{L}$ samples on patterned detection zones. B. Luminescent image from the CMOS sensor, with triplicate DBAE samples, concentrations labeled below. C. A surface intensity plot shows the detection zones where luminescent intensity is integrated for each DBAE concentration. All concentrations labeled are in $\mu \mathrm{M}$.
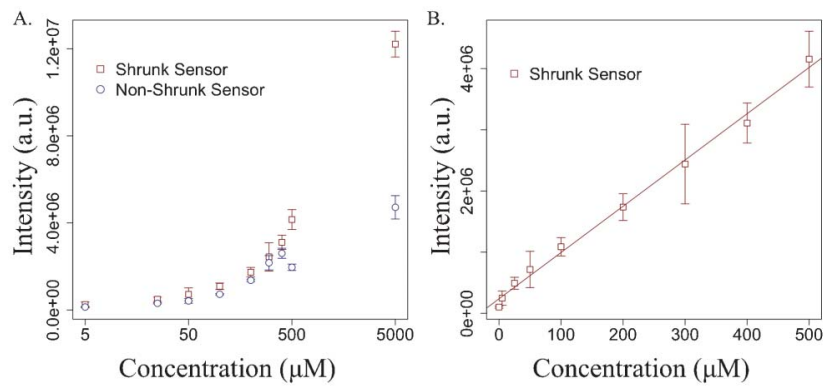

Fig. 5 Concentration curves comparing planar and wrinkled electrode sensors. A. The full DBAE concentration range is shown on a log scale. B. The linear region of the concentration curve.

PO films are shrunk using this approach, the bare polymer surface is flat and smooth (Supplementary Fig. S1B十). This method provides the possibility for integration with microfluidics where a plastic top-sheet with microfluidic channels can be thermally bonded to the electrode layer. ${ }^{21}$ In this demonstration, the first method was chosen for the ability to prevent any surface wetting of samples outside the patterned Au detection zones of our ECL sensor.

To show the utility and performance gains of these electrodes in a POC scenario, an integrated microdevice for ECL detection with an inexpensive digital camera was fabricated. Au patterned thin films on non-shrunk PO films served as a planar electrode control. Results for these $\mathrm{Au}$ wrinkled thin film electrode devices (Fig. 4) showed detectable signals across the entire tested concentration range and a linear regime from $5-500 \mu \mathrm{M}$ and a LOD of $1.7 \mu \mathrm{M}$ (Fig. 5). This LOD is more than two orders of magnitude lower than similar paper-based ECL systems in the literature ${ }^{14}$ which also utilizes a consumer grade camera. The enhancement of electrochemical activity in the wrinkled electrodes is seen markedly at the higher end of the concentration range (Fig. 5A). This is expected because the increased surface area of the wrinkled electrode increases the rate of electron transfer into the electrolytic solution for faster oxidation of the ECL complex. This translates to more luminescence when integrated over time by the CMOS camera sensor. The advantages of high surface area become less apparent at the lowest concentrations when the reaction rate is limited by the diffusion of DBAE to the electrode surface.

\section{Conclusions}

We present an integrated plastic ECL sensor with shrinkinduced high surface area electrodes using standard, scalable microfabrication techniques with commodity shrink-wrap film. Wrinkled $\mathrm{Au}$ thin film electrodes produced by thermally shrinking integrated shape-memory PO not only induces high surface area, but also, improves robustness and electrical properties. Integration of PO with standard micromachining processes provides reliable, higher resolution patterning as well as manufacturing scalability. Notably, with a 20-fold increase in resolution from shrinking, we are able to achieve 
higher resolution than with photolithography alone or any other previously reported methods using shape-memory polymers. In comparing planar and wrinkled thin film electrodes, wrinkled thin film electrodes show larger detectable signals, better range and sensitivity, and a LOD two magnitudes lower than similar existing systems. ${ }^{14}$ These electrodes, integrated with a flexible thermoplastic substrate, are suitable for use with microfluidics or as conformal electrodes as the plastic is bendable and moldable. These attributes make this technical innovation easily adapted for POC applications.

\section{Acknowledgements}

This work was supported in part by the NIH New Innovator Award (1DP2OD007283) and in part by the Defense Advanced Research Projects Agency (DARPA) N/MEMS S\&T Fundamentals Program under grant no. N66001-1-4003 issued by the Space and Naval Warfare Systems Center Pacific (SPAWAR) to the Micro/nano Fluidics Fundamentals Focus (MF3) Center.

\section{References}

1 M. Urdea, L. A. Penny, S. S. Olmsted, M. Y. Giovanni, P. Kaspar, A. Shepherd, P. Wilson, C. A. Dahl, S. Buchsbaum, G. Moeller and D. C. Hay Burgess, Nature, 2006, 444, 73-9.

2 P. Yager, G. J. Domingo and J. Gerdes, Annu. Rev. Biomed. Eng., 2008, 10, 107-44.

3 H. Sharma, D. Nguyen, A. Chen, V. Lew and M. Khine, Ann. Biomed. Eng., 2011, 39, 1313-27.

4 C. D. Chin, T. Laksanasopin, Y. K. Cheung, D. Steinmiller, V. Linder, H. Parsa, J. Wang, H. Moore, R. Rouse, G. Umviligihozo, E. Karita, L. Mwambarangwe, S. L. Braunstein, J. van de Wijgert, R. Sahabo, J. E. Justman, W. El-Sadr and S. K. Sia, Nat. Med., 2011, 17, 1015-9.

5 C. D. Chin, V. Linder and S. K. Sia, Lab Chip, 2012, 12, 2118-34.

6 X. Li, D. R. Ballerini and W. Shen, Biomicrofluidics, 2012, 6, 11301-1130113.

7 L. Ge, J. Yan, X. Song, M. Yan, S. Ge and J. Yu, Biomaterials, 2012, 33, 1024-31.

8 C. Qiu, X. Wang, X. Liu, S. Hou and H. Ma, Electrochim. Acta, 2012, 67, 140-146.

9 N. J. Ronkainen, H. B. Halsall and W. R. Heineman, Chem. Soc. Rev., 2010, 39, 1747-63.
10 C.-L. Sun, H.-H. Lee, J.-M. Yang and C.-C. Wu, Biosens. Bioelectron., 2011, 26, 3450-5.

11 H. Wei and E. Wang, Luminescence, 2011, 26, 77-85.

12 Y. Yuan, S. Han, L. Hu, S. Parveen and G. Xu, Electrochim. Acta, 2012, 82, 484-492.

13 E. J. O'Reilly, T. E. Keyes, R. J. Forster and L. Dennany, Analyst, 2013, 138, 677-82.

14 J. L. Delaney, C. F. Hogan, J. Tian and W. Shen, Anal. Chem., 2011, 83, 1300-6.

15 N. P. Sardesai, K. Kadimisetty, R. Faria and J. F. Rusling, Anal. Bioanal. Chem., 2013, 405, 3831-8.

16 D. Nawarathna, N. Norouzi, J. McLane, H. Sharma, N. Sharac, T. Grant, A. Chen, S. Strayer, R. Ragan and M. Khine, Appl. Phys. Lett., 2013, 102, 63504.

17 D. Nguyen, S. Sa, J. D. Pegan, B. Rich, G. Xiang, K. E. McCloskey, J. O. Manilay and M. Khine, Lab Chip, 2009, 9, 3338-44.

18 D. Nguyen, J. McLane, V. Lew, J. Pegan and M. Khine, Biomicrofluidics, 2011, 5, 22209.

19 A. Chen, D. K. Lieu, L. Freschauf, V. Lew, H. Sharma, J. Wang, D. Nguyen, I. Karakikes, R. J. Hajjar, A. Gopinathan, E. Botvinick, C. C. Fowlkes, R. A. Li and M. Khine, Adv. Mater., 2011, 23, 5785-91.

20 S. Jayadev, J. Pegan, D. Dyer, J. McLane, J. Lim and M. Khine, Smart Mater. Struct., 2013, 22, 014014.

21 D. Taylor, D. Dyer, V. Lew and M. Khine, Lab Chip, 2010, 10, 2472-5.

22 H. Sharma, D. Nguyen, A. Chen, V. Lew and M. Khine, Ann. Biomed. Eng., 2011, 39, 1313-27.

23 C. M. Gabardo, Y. Zhu, L. Soleymani and J. M. MoranMirabal, Adv. Funct. Mater., 2013, 23, 3030-3039.

24 F. Greco, L. Ventrelli, P. Dario, B. Mazzolai and V. Mattoli, Int. J. Hydrogen Energy, 2012, 37, 17529-17539.

25 L. Soleymani, Z. Fang, E. H. Sargent and S. O. Kelley, Nat. Nanotechnol., 2009, 4, 844-8.

26 K. B. Cederquist and S. O. Kelley, Curr. Opin. Chem. Biol., 2012, 16, 415-21.

27 D. Nguyen, D. Taylor, K. Qian, N. Norouzi, J. Rasmussen, S. Botzet, M. Lehmann, K. Halverson and M. Khine, Lab Chip, 2010, 10, 1623-6.

28 L. Xue, L. Guo, B. Qiu, Z. Lin and G. Chen, Electrochem. Commun., 2009, 11, 1579-1582.

29 C. A. Schneider, W. S. Rasband and K. W. Eliceiri, Nat. Methods, 2012, 9, 671-675.

30 L. R. Freschauf, J. McLane, H. Sharma and M. Khine, PLoS One, 2012, 7, e40987.

31 K. L. Chopra, L. C. Bobb and M. H. Francombe, J. Appl. Phys., 1963, 100, 4-7. 